\title{
The Impact of Advanced Image-Guided Breast Surgery and Oncoplastic Techniques on Margin Positivity in Breast Conserving Surgery
}

\author{
Tolga Ozmen ${ }^{1}$, Eli Avisar ${ }^{1}$ \\ 1. Surgical Oncology, University of Miami, Miller School of Medicine, Miami, USA
}

Corresponding author: Tolga Ozmen, drtolgaozmen@yahoo.com.tr

\section{Abstract \\ Objective}

Positive margins remain a significant psychological and economic burden after breast conserving surgery. The aim of this study was to test the hypothesis that advanced oncoplastic techniques as well as intraoperative integrative imaging with intraoperative ultrasound and mobile digital specimen radiography decreases positive margin rate in breast conserving surgery.

\section{Methods}

A single-institution retrospective review of a prospectively collected database was performed. Patients with breast neoplasms who underwent lumpectomy with or without using intraoperative integrative imaging approaches and oncoplastic techniques were included. The primary outcome was positive margin rate for each technique.

\section{Results}

A total of 392 patients were included in the study. The median age of the cohort was 59 years. Overall positive margin rate was $15 \%$. Ductal carcinoma in situ (DCIS) histology and larger tumor size were associated with higher positive margin rate. Intraoperative integrative imaging significantly decreased positive margin rate $(9 \%$ vs. $18 \%, \mathrm{p}=0.018)$. Oncoplastic techniques also decreased positive margin rate from $16 \%$ to $12 \%$, however this was not significant.

\section{Conclusion}

Positive margin rate was significantly lower when intraoperative integrative imaging was used. Oncoplastic techniques also decreased positive margin rate in a selected group of patients with large tumor size. We suggest incorporating these techniques in all breast conserving surgery cases.

Review began $11 / 08 / 2020$ Review ended 11/25/2020 Published 12/01/2020

\section{() Copyright 2020}

Ozmen et al. This is an open access article distributed under the terms of the Creative Commons Attribution License CC-BY 4.0., which permits unrestricted use, distribution, and reproduction in any medium, provided the original author and source are credited.
Categories: General Surgery, Oncology

Keywords: breast cancer, image-guided surgery, resection margins, surgical margins, residual disease, ultrasound

\section{Introduction}

Breast cancer is the most commonly diagnosed cancer in women with an incidence rate of $13 \%$ [1]. The treatment of this common cancer has evolved significantly from radical mastectomy to breast conserving surgery (BCS) [2,3]. BCS offers a significant benefit for many patients by preserving body image, saving breast mound and sensation. These advantages come with a price of increased positive surgical margin requiring additional surgery. Positive margin rate (PMR) is reported as high as $40 \%$ in the literature [4-8]. In North America, $25 \%$ of patients undergo a re-excision surgery due margin positivity after BCS [9]. Decrease in PMR has become a major research focus leading to major technologic, surgical and research investments. In addition, significant efforts have been made in an attempt to develop consensus and guidelines in the definition and management of positive margin.

A variety of techniques have been used to localize non-palpable breast cancers including wires, radioactive and non-radioactive seeds, and intra-operative ultrasound (IOUS). Besides localizing the lesion, IOUS can also be used to guide the resection margins in both palpable and non-palpable breast cancers. Some studies claim one technique being superior to another, however the data are conflicting and the gold standard technique is yet to be determined [10-13]. In 2015, the American Society of Breast Surgeons reviewed all the available evidence and developed a toolbox of recommendations to reduce PMR and improve cosmetic outcome after BCS. They concluded that evidence was not definitive to recommend a single technique [14].

For needle localized tumors, the standard approach is to confirm resection of the targeted lesion with specimen radiography. Conventional specimen radiography requires the specimen to be transported to 
radiology department, while the patient stays in the operating room under anesthesia. Prolonged anesthesia time increases both anesthesia associated morbidity, mortality and the cost associated with operating room occupation [15]. Mobile digital specimen radiography (MDSR), first introduced in 1998, is a mobile X-ray device, allowing specimen images to be obtained in the operating room and interpreted remotely, which bypasses transportation of the specimen. Studies showed that despite lower image quality, MDSR allows verification of target lesion excision in confidence [15-17].

Excising a larger volume of tissue by implementing oncoplastic techniques (OT) also has the potential to decrease PMR without compromising the cosmetic outcome [18]. OT were also included into the American Society of Breast Surgeons' toolbox of recommendations and the consensus strongly recommended it to be applied for patients with large tumors undergoing BCS [14].

Above mentioned integrated intraoperative imaging (III) techniques and OT are commonly used for patients undergoing breast surgery in our clinic. The aim of this study was to test the effects of III and OT on PMR and to share our experience on margin positivity.

\section{Materials And Methods}

This is a retrospective analysis of a prospectively collected database. We queried all women treated in our clinic for invasive ductal cancer (IDC), invasive lobular carcinoma (ILC), ductal carcinoma in situ (DCIS) or Phyllodes tumor between 2010 and 2015. The data included demographics and tumor characteristics as age, histological tumor type, tumor volume and specimen volume as well as surgical data regarding use of OT and/or III and margin positivity. This study was approved by our institutional review board (\#20053311) who granted a waiver of consent. The study was compliant with the Health Insurance Portability and Accountability Act (HIPAA).

During the course of data collection, we experienced a gradual incorporation of MDSR, IOUS, and OT in the operating room among a homogenous and well-trained group of surgeons thus enabling a rare opportunity to compare these techniques with conventional methods in a random, non-tumor and non-patient related fashion.

\section{Definition of terms}

Surgeries as flap advancement, breast reduction, mastopexy or adjacent breast tissue advancement were accepted as OT. MDSR and intraoperative IOUS were accepted as III modalities.

During the course of the study, negative margins were defined at our institution as "no tumor on ink" for both invasive and non-invasive cancer. This is in accordance with the subsequently published Society of Surgical Oncology (SSO) and the American Society for Radiation Oncology (ASTRO) consensus guidelines for surgical margins for invasive cancer [19] but slightly more lenient than the later published for DCIS [20]. For Phyllodes tumor, we aimed to excise the tumor with wide local excision (>10mm). The decision to proceed with re-excision for positive margins was made on a case per case basis and relied on patient and tumor factors as well as a multidisciplinary discussion.

\section{Statistical analysis}

Statistical analysis was carried out using Statistical Package for Social Sciences (SPSS) Statistics version 23 (IBM Corp., Armonk, NY, USA). Student's T-test was used to compare continuous values, while chisquare test was used for categorical values. A "p" value less than 0.05 was considered statistically significant.

\section{Results}

A total of 392 patients were included in the study. The median age was 59 (27-92) years. PMR was $15 \%$. Invasive ductal carcinoma was the most common histologic tumor type (78\%) followed by DCIS (13\%), invasive lobular carcinoma (5\%) and Phyllodes tumor (4\%). OT and III were used in 36\% and 32\% of the cases, respectively. MDSR was the most commonly used III modality (17\%), followed by IOUS (12\%). Median excised tumor volume was $3.4(0.2-1701.1) \mathrm{cm}^{3}$ and median excised specimen volume was $166.5(1.3-1802.2)$ $\mathrm{cm}^{3}$ (Table 1). 


\section{Cureus}

\begin{tabular}{|lll|}
\hline Age (y) & & $59(27-92)$ \\
\hline Positive surgical margin(s) & & $58(15)$ \\
\hline Oncoplastic technique & None & $142(36)$ \\
\hline Integrated intraoperative imaging & Intraoperative ultrasound & $265(68)$ \\
& MDSR & $47(12)$ \\
\hline Histologic type of tumor & Both & $66(17)$ \\
& Invasive ductal carcinoma & $14(3)$ \\
\hline Volume of tumor $\left(\mathrm{cm}^{3}\right)$ & Ductal carcinoma in-situ & $306(78)$ \\
\hline Volume of specimen $\left(\mathrm{cm}^{3}\right)$ & Invasive lobular carcinoma & $49(13)$ \\
\hline
\end{tabular}

\section{TABLE 1: Descriptive parameters of the cohort}

Categorical variables are presented as $\mathrm{n}(\%)$

Continuous variables are presented as Median [Range]

åMDSR: mobile digital specimen radiography

In our cohort, patients who underwent OT had significantly larger specimen volume $\left(235.2 \mathrm{~cm}^{3} \mathrm{vs} .188 .2\right.$ $\left.\mathrm{cm}^{3}, \mathrm{p}=0.024\right)$. Also, the mean age of patients in the OT group was younger, but this was statistically not significant (56.7 vs. 58.6, $\mathrm{p}=0.077$ ). III was most commonly used in DCIS patients (44\%). A histology of DCIS $(31 \%, \mathrm{p}=0.002)$ and larger tumor volume $\left(51.4 \mathrm{~cm}^{3}\right.$ vs. $\left.10.6 \mathrm{~cm}^{3}, \mathrm{p}=0.002\right)$ were associated with higher margin positivity. III decreased positive margin risk by $56 \%$ ( $9 \%$ vs. $18 \%$; $\mathrm{p}=0.018$, odds ratio [OR] 0.44 [0.22-0.88]) (Table 2). 


\section{Cureus}

\begin{tabular}{|c|c|c|c|c|c|c|c|}
\hline & & \multicolumn{2}{|c|}{ Oncoplastic Technique } & \multirow{2}{*}{$\mathbf{P}$} & \multicolumn{2}{|c|}{ Integrated Intraoperative Imaging } & \multirow{2}{*}{$\mathbf{P}$} \\
\hline & & No & Yes & & No & Yes & \\
\hline \multicolumn{2}{|l|}{ Age (y) } & $58.6 \pm 9.8$ & $56.7 \pm 10$ & 0.077 & $58.1 \pm 10.3$ & $57.6 \pm 9.1$ & 0.695 \\
\hline \multirow{4}{*}{ Histologic type of tumor } & Invasive ductal carcinoma & $189(62)$ & $117(38)$ & \multirow{4}{*}{0.128} & $200(65)$ & $106(35)$ & \multirow{4}{*}{0.032} \\
\hline & Ductal carcinoma in-situ & $33(67)$ & $16(33)$ & & $32(56)$ & $17(44)$ & \\
\hline & Invasive lobular carcinoma & $13(68)$ & $6(32)$ & & $15(79)$ & $4(21)$ & \\
\hline & Phyllodes tumor & $15(88)$ & $2(12)$ & & $17(100)$ & $0(0)$ & \\
\hline \multicolumn{2}{|l|}{ Volume of tumor $\left(\mathrm{cm}^{3}\right)$} & $16.4 \pm 108.5$ & $17 \pm 49.4$ & 0.937 & $19.9 \pm 110.6$ & $9.8 \pm 17.1$ & 0.148 \\
\hline \multicolumn{2}{|l|}{ Volume of specimen $\left(\mathrm{cm}^{3}\right)$} & $188.2 \pm 183.7$ & $235.2 \pm 204.3$ & 0.024 & $196.4 \pm 188.9$ & $223.7 \pm 199.1$ & 0.199 \\
\hline
\end{tabular}

TABLE 2: Comparison of descriptive parameters with oncoplastic techniques and integrated intraoperative imaging.

Categorical variables are presented as $\mathrm{n}(\%)$

Continuous variables are presented as Mean $\pm \mathrm{SD}$

In multivariate analysis, DCIS histology and larger tumor volume continued to be statistically significant, however, p value for III was 0.052 (Table 3). 


\section{Cureus}

\begin{tabular}{|c|c|c|c|c|c|}
\hline & & \multicolumn{2}{|l|}{ Margin Status } & \multirow{2}{*}{ Univariate $p$} & \multirow{2}{*}{ Multivariate $p$} \\
\hline & & Negative & Positive & & \\
\hline \multicolumn{2}{|l|}{ Age(y) } & $57.9 \pm 10.1$ & $57.8 \pm 9.3$ & 0.942 & \\
\hline \multirow{4}{*}{ Histologic type of tumor } & Invasive ductal carcinoma & $272(89)$ & 34 (11) & \multirow{4}{*}{0.002} & \multirow{4}{*}{0.038} \\
\hline & Ductal carcinoma in-situ & $34(69)$ & $15(31)$ & & \\
\hline & Invasive lobular carcinoma & $15(79)$ & $4(21)$ & & \\
\hline & Phyllodes tumor & $12(71)$ & $5(29)$ & & \\
\hline \multicolumn{2}{|l|}{ Volume of tumor $\left(\mathrm{cm}^{3}\right)$} & $10.6 \pm 23.9$ & $51.4 \pm 229.6$ & 0.002 & 0.015 \\
\hline \multicolumn{2}{|l|}{ Volume of specimen $\left(\mathrm{cm}^{3}\right)$} & $198.6 \pm 158.1$ & $243.3 \pm 326.3$ & 0.103 & \\
\hline \multirow{2}{*}{ OT $^{*}$} & No & $209(84)$ & $41(16)$ & \multirow{2}{*}{0.235} & \\
\hline & Yes & $125(88)$ & $17(12)$ & & \\
\hline \multirow{2}{*}{$\mathrm{III}^{\mathrm{w}}$} & No & $218(82)$ & $47(18)$ & \multirow{2}{*}{0.018 OR 0.44 [0.22-0.88] } & \multirow{2}{*}{0.052} \\
\hline & Yes & $116(91)$ & $11(9)$ & & \\
\hline \multirow{4}{*}{ IIII type } & No & $218(82)$ & $47(18)$ & \multirow{4}{*}{0.088} & \\
\hline & MDSR $^{a}$ & $59(89)$ & $7(11)$ & & \\
\hline & Intraoperative US & $43(91)$ & $4(9)$ & & \\
\hline & Both & $14(100)$ & $0(0)$ & & \\
\hline
\end{tabular}

\section{TABLE 3: Parameters affecting with margin status}

Categorical variables are presented as $\mathrm{n}(\%)$, continuous variables are presented as Mean \pm SD

${ }^{*} \mathrm{OT}$ = oncoplastic technique;

wIII = integrated intraoperative imaging

åMDSR: mobile digital specimen radiography

In an attempt to better understand the specific contributions of OT and III to the margin status, we analyzed the effect of III on PMR after stratification into OT vs. no OT. In the "no OT" group, III significantly decreased PMR ( $0 \%$ vs. $18 \%$, p=0.026, OR 0.82 [0.77-0.87]). In the "OT" group, III did decrease PMR by $5 \%$, but this difference was not statistically significant (Table 4). 


\section{Cureus}

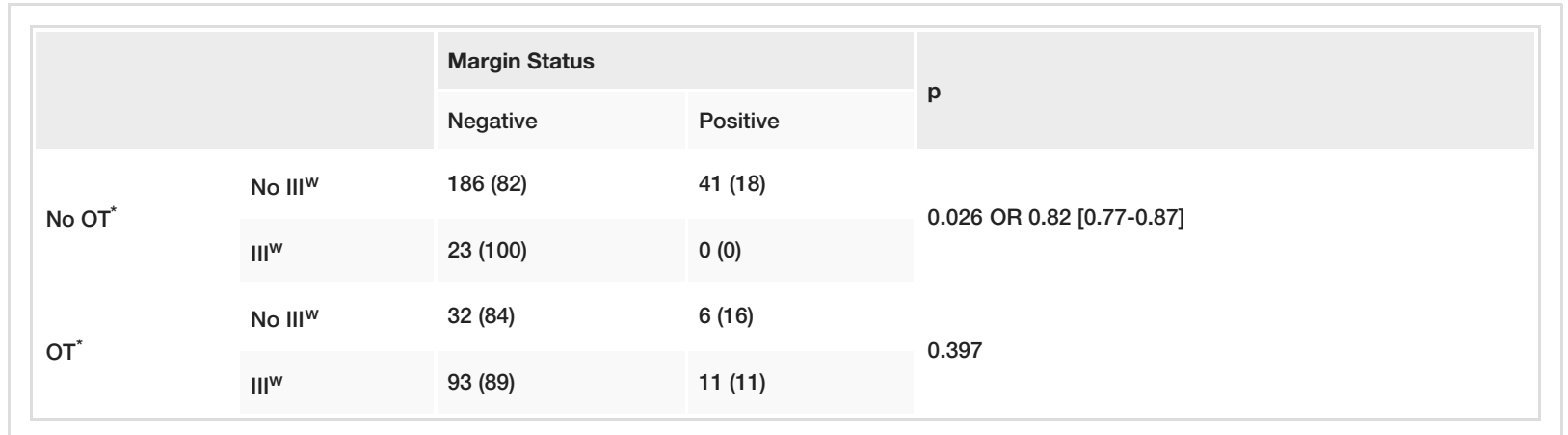

TABLE 4: Effect of III on margin status after stratification by OT

Data are presented as $\mathrm{n}(\%)$,

${ }^{*} \mathrm{OT}=$ oncoplastic technique;

$\mathrm{w}_{\mathrm{III}}=$ integrated intraoperative imaging

\section{Discussion}

The wide implementation and acceptance of breast cancer screening programs coupled with significant advancements in imaging technology have resulted in a large increase in early breast cancer diagnosis, making BCS the preferred treatment option. The many advantages of breast conservation are beyond the scope of this discussion. BCS, however, is also associated with an inherent significant risk of margin positivity. In the literature, PMR has been reported as high as 40\% [4-8]. Positive margins are frustrating to patients and providers alike and are associated with an increase in re-operations, mastectomy rates, medical and radiation treatments all of which are difficult for the patients and expensive for the health care systems. Various techniques have been proposed in an attempt to minimize the PMR with the best approach yet to be determined. In our series, we calculated a PMR of $15 \%$. This rate is slightly lower than the $20-40 \%$ PMR range reported in the literature [21,22]. Previous studies have shown that DCIS histology is associated with an increase in PMR [23-25]. Similarly, in our study, PMR was higher among DCIS patients. Another parameter we found to be associated with a higher margin positivity was larger tumor size. This finding was also previously reported by Singletary et al. [7].

In our study, III decreased PMR risk by 56\%. The effect of MDSR on PMR has been previously studied and was shown to decrease margin positivity by $60-96 \%[25,26]$. Versteegden et al. reported in their study that the benefit of radiography was however weakened in in-situ histology, and this was attributed to the extent of disease becoming harder to define in these cases [27]. Karanlik reported an 11\% decrease and Krekel reported a 13\% decrease in PMR with the use of IOUS for BCS [10,28]. In our study, IOUS decreased PMR to a greater extent than MDSR and the combination of MDSR and IOUS yielded the lowest PMR. We also looked at invasive and in-situ carcinoma separately. In invasive ductal cancer, MDSR alone and IOUS alone decreased PMR similarly ( $8 \%$ and $9 \%$ vs. 13\% respectively). However, the lowest PMR was achieved when MDSR and IOUS were used together ( $0 \%$ vs. $13 \%$ ). In in-situ disease, MDSR alone decreased PMR from $38 \%$ to $21 \%$ and none of the patients underwent IOUS alone in this histology subtype. Once again, the lowest PMR was achieved when MDSR and IOUS were used together (0\% vs. 38\%). It would appear therefore, that MDSR and IOUS should be used together to achieve the lowest PMR in both invasive and in-situ disease. However, we believe that this should be tested in larger cohorts.

As already mentioned in the introduction, the definition of PMR for in-situ disease was no tumor on ink which is different than the later published recommendation of $2 \mathrm{~mm}$ margins. No tumor on ink however, was the accepted definition of PMR in all NSABP studies on in-situ disease and still has a very significant validity. In fact, although the current guidelines recommend a $2 \mathrm{~mm}$ margins for in-situ disease, the need for re-excision is left vague as long as no tumor is present on ink. Additional considerations as tumor histology, patient age, additional treatment modalities etc. have to be considered in those cases. Furthermore, the current management of in-situ breast neoplasms continues to be controversial when on one hand wider margins are recommended and on the other hand less surgery and less radiation are been actively studied including observation only. In regard to our series, given the fashion of the data recording and collection as well as the current practice at the time of this study it would have not been feasible to redefine positive margins for in-situ disease. We believe however that the data on PMR as we defined it is still valid and relevant. Potentially, MDSR and IOUS could be similarly used after adoption of wider margins and being associated with similar results, however this will need further studies.

As the tumor size increases, achieving negative margins along with a successful cosmetic result becomes more challenging. To that end, OT can be of great help to the operating surgeon. In the American Society of 
Breast Surgeons recommendation toolbox, OT was defined as having the potential to decrease PMR by allowing a larger specimen to be excised [17]. Specimen volume excised in the OT group was significantly larger in our study and indeed the PMR in this group was lower by $4 \%$. The decrease in PMR was more prominent when OT was used in larger tumors suggesting that those cases benefit most from the addition of OT to III.

Since both OT and III decrease PMR, it is not surprising that in the multivariate analysis III lost some of its efficacy. Its beneficial effect on PMR is however still significant enough so that it barely missed the 0.05 significance. Clearly a larger sample might help demonstrate that strong trend. In the subgroup without use of OT a sharp and statistically significant PMR decrease was easily observed. Different techniques have been described in an attempt to decrease PMR for BCS. One of them, shave cavity margins, have demonstrated a $50 \%$ decrease in PMR [7] but the cosmetic results are impacted by removal of additional margins that might not be involved. Additionally, the increase in pathology samples and cost are significant. Other techniques require use of specific instruments meant to identify the positive margins in the operating room. Most of those tools, however, are expensive, add operating time, and are associated with losing specificity in the pursuit of sensitivity. In this study we have demonstrated that PMR can be decreased by roughly $50 \%$ and brought down to around $10 \%$ by simple use of technology already available and without compromising breast cosmesis.

Some of the limitations of this study include the relatively small sample size and the fact that it is a retrospective analysis. For those reasons further subset analysis is difficult and we cannot correct for possible time variation as, specific surgeons learning curves as well as surgeon-specific factors. It should be mentioned however that the same group of surgeons participated in the whole study and previous QA projects revealed no significant differences in positive margin rates between surgeons. Prospective randomized studies and larger sample size will be necessary to better assess the effects of III and OT on PMR.

\section{Conclusions}

Integrated intraoperative imaging significantly decreases PMR using readily available and inexpensive imaging tools and without additional tissue resection. OT enables better cosmetic results for patients with larger tumor volumes with no compromise of the PMR.

\section{Additional Information \\ Disclosures}

Human subjects: Consent was obtained by all participants in this study. University of Miami, Miller School of Medicine institutional review board issued approval 20053311. This project was approved by the University of Miami, Miller School of Medicine institutional review board (IRB \#20053311). Animal subjects: All authors have confirmed that this study did not involve animal subjects or tissue. Conflicts of interest: In compliance with the ICMJE uniform disclosure form, all authors declare the following: Payment/services info: All authors have declared that no financial support was received from any organization for the submitted work. Financial relationships: All authors have declared that they have no financial relationships at present or within the previous three years with any organizations that might have an interest in the submitted work. Other relationships: All authors have declared that there are no other relationships or activities that could appear to have influenced the submitted work.

\section{References}

1. Breast Cancer Awareness Month in October . (2012). http://www.who.int/cancer/events/breast_cancer_month/en/.

2. Veronesi U, Cascinelli N, Mariani L, et al.: Twenty-year follow-up of a randomized study comparing breastconserving surgery with radical mastectomy for early breast cancer. N Engl J Med. 2002, 347:1227-1232. 10.1056/NEJMoa020989

3. Fisher B, Anderson S, Bryant J, et al.: Twenty-year follow-up of a randomized trial comparing total mastectomy, lumpectomy, and lumpectomy plus irradiation for the treatment of invasive breast cancer. $\mathrm{N}$ Engl J Med. 2002, 347:1233-1241. 10.1056/NEJMoa022152

4. Volders JH, Haloua MH, Krekel NM, Meijer S, van den Tol PM: Current status of ultrasound-guided surgery in the treatment of breast cancer. World J Clin Oncol. 2016, 7:44-53. 10.5306/wjco.v7.i1.44

5. Coopey S, Smith BL, Hanson S, Buckley J, Hughes KS, Gadd M, Specht MC: The safety of multiple reexcisions after lumpectomy for breast cancer. Ann Surg Oncol. 2011, 18:3797-3801. 10.1245/s10434-011$1802-4$

6. Krekel N, Zonderhuis B, Muller S, et al.: Excessive resections in breast-conserving surgery: a retrospective multicentre study. Breast J. 2011, 17:602-609. 10.1111/j.1524-4741.2011.01198.x

7. Singletary SE: Surgical margins in patients with early-stage breast cancer treated with breast conservation therapy. Am J Surg. 2002, 184:383-393. 10.1016/s0002-9610(02)01012-7

8. Krekel NM, Zonderhuis BM, Stockmann HB, et al.: A comparison of three methods for nonpalpable breast cancer excision. Eur J Surg Oncol. 2011, 37:109-115. 10.1016/i.ejso.2010.12.006

9. Wilke LG, Czechura T, Wang C, Lapin B, Liederbach E, Winchester DP, Yao K: Repeat surgery after breast conservation for the treatment of stage 0 to II breast carcinoma: a report from the National Cancer Data 
Base, 2004-2010. JAMA Surg. 2014, 149:1296-1305. 10.1001/jamasurg.2014.926

10. Karanlik H, Ozgur I, Sahin D, Fayda M, Onder S, Yavuz E: Intraoperative ultrasound reduces the need for reexcision in breast-conserving surgery. World J Surg Oncol. 2015, 13:321. 10.1186/s12957-015-0731-2

11. Olsha O, Shemesh D, Carmon M, Sibirsky O, Abu Dalo R, Rivkin L, Ashkenazi I: Resection margins in ultrasound-guided breast-conserving surgery. Ann Surg Oncol. 2011, 18:447-452. 10.1245/s10434-010-12800

12. Keller MD, Majumder SK, Kelley MC, Meszoely IM, Boulos FI, Olivares GM, Mahadevan-Jansen A: Autofluorescence and diffuse reflectance spectroscopy and spectral imaging for breast surgical margin analysis. Lasers Surg Med. 2010, 42:15-23. 10.1002/1sm.20865

13. Allweis TM, Kaufman Z, Lelcuk S, et al.: A prospective, randomized, controlled, multicenter study of a realtime, intraoperative probe for positive margin detection in breast-conserving surgery. Am J Surg. 2008, 196:483-489. 10.1016/j.amjsurg.2008.06.024

14. Landercasper J, Attai D, Atisha D, et al.: Toolbox to reduce lumpectomy reoperations and improve cosmetic outcome in breast cancer patients: the American Society of Breast Surgeons Consensus Conference. Ann Surg Oncol. 2015, 22:3174-3183. 10.1245/s10434-015-4759-X

15. Wang Y, Ebuoma L, Saksena M, Liu B, Specht M, Rafferty E: Clinical evaluation of a mobile digital specimen radiography system for intraoperative specimen verification. AJR Am J Roentgenol. 2014, 203:457-462. 10.2214/AJR.13.11408

16. Kaufman CS, Bachman BA, Jacobson L, Kaufman LB, Mahon C, Gambrell L: Intraoperative digital specimen mammography: prompt image review speeds surgery. Am J Surg. 2006, 192:513-515. 10.1016/j.amjsurg.2006.06.022

17. Kaufman CS, Jacobson L, Bachman BA, et al.: Intraoperative digital specimen mammography: rapid, accurate results expedite surgery. Ann Surg Oncol. 2007, 14:1478-1485. 10.1245/s10434-006-9126-5

18. Ozmen T, Polat AV, Polat AK, Bonaventura M, Johnson R, Soran A: Factors affecting cosmesis after breast conserving surgery without oncoplastic techniques in an experienced comprehensive breast center. Surgeon. 2015, 13:139-144. 10.1016/j.surge.2013.12.005

19. Buchholz TA, Somerfield MR, Griggs JJ, et al.: Margins for breast-conserving surgery with whole-breast irradiation in stage I and II invasive breast cancer: American Society of Clinical Oncology endorsement of the Society of Surgical Oncology/American Society for Radiation Oncology consensus guideline. J Clin Oncol. 2014, 32:1502-1506. 10.1200/JCO.2014.55.1572

20. Morrow M, Van Zee KJ, Solin LJ, et al.: Society of Surgical Oncology-American Society for Radiation Oncology-American Society of Clinical Oncology consensus guideline on margins for breast conserving surgery with whole breast irradiation in ductal carcinoma in situ. J Clin Oncol. 2016, 34:4040-4046. 10.1200/JCO.2016.68.3573

21. McCahill LE, Single RM, Aiello Bowles EJ, et al.: Variability in reexcision following breast conservation surgery. JAMA. 2012, 307:467-475. 10.1001/jama.2012.43

22. Chagpar AB, Killelea BK, Tsangaris TN, et al.: A randomized, controlled trial of cavity shave margins in breast cancer. N Engl J Med. 2015, 6:503-510. 10.1056/NEJMoa1504473

23. Miller AR, Brandao G, Prihoda TJ, Hill C, Cruz AB Jr, Yeh IT: Positive margins following surgical resection of breast carcinoma: analysis of pathologic correlates. J Surg Oncol. 2004, 86:134-140. 10.1002/jso.20059

24. Wazer DE, Schmidt-Ullrich RK, Ruthazer R, DiPetrillo T, Boyle T, Kanski J, Safaii H: The influence of age and extensive intraductal component histology upon breast lumpectomy margin assessment as a predictor of residual tumor. Int J Radiat Oncol Biol Phys. 1999, 45:885-891. 10.1016/S0360-3016(99)00196-0

25. McCormick JT, Keleher AJ, Tikhomirov VB, Budway RJ, Caushaj PF: Analysis of the use of specimen mammography in breast conservation therapy. Am J Surg. 2004, 188:433-436.

10.1016/j.amjsurg.2004.06.030

26. Bathla L, Harris A, Davey M, Sharma P, Silva E: High resolution intra-operative two-dimensional specimen mammography and its impact on second operation for re excision of positive margins at final pathology after breast conservation surgery. Am J Surg. 2011, 202:387-394. 10.1016/j.amjsurg.2010.09.031

27. Versteegden DA, Keizer LG, Schlooz-Vries MS, Duijm LM, Wauters CP, Strobbe LA: Performance characteristics of specimen radiography for margin assessment for ductal carcinoma in situ: a systematic review. Breast Cancer Res Treat. 2017, 166:669-679. 10.1007/s10549-017-4475-2

28. Krekel NM, Haloua MH, Lopes Cardozo AM, et al.: Intraoperative ultrasound guidance for palpable breast cancer excision (COBALT trial): a multicenter, randomized controlled trial. Lancet Oncol. 2013, 14:48-54. 10.1016/S1470-2045(12)70527-2 